\title{
Impact of Neoadjuvant Chemo Radiation in Carcinoma Rectum: A Tertiary Care Institute Experience
}

\author{
Pramod Kumar Singh*, Rajit Rattan, Rakesh Kapoor, Ritesh Kumar and Ravi Teja \\ Department of radiotherapy, Jk cancer institute, India
}

Submission: July 17, 2018; Published: October 26, 2018

"Correspondence Address: Pramod Kumar Singh, Department of radiotherapy, Jk cancer institute, Kanpur Uttar Pradesh, India, Email: drpramodsingh16@gmail.com

\begin{abstract}
Purpose: To evaluate the rate of pathologic complete response (pCR) and the toxicity ofneoadjuvant chemoradiotherapy (chemoRT) regimens for Stage T3-T4 rectal cancer.

Methods and Materials: Patients with Stage T3 or T4 rectal cancer of $<12 \mathrm{~cm}$ from the anal verge were randomized to preoperative RT (45 Gy in 25 fractionsover 5 weeks in 1.8 Gy per fractions) with concurrent chemotherapy (5FU + levcoverine). Surgery was performed 4-8 weeks after chemoRT, and adjuvant chemotherapy $4-6$ weeks after surgery.

Results: A total of 25 patients are analyzed. The median age at diagnosis was 52.00 years. 23 of the patients were male, while 2 of them were female. The median 3-year disease free survival was 50\%. Pathologic complete response (pCR) was $12 \%$ and the preoperative chemo RT rate of Grade 3-4 hematologic toxicity was $9 \%$ and $4 \%$ and the rate of Grade 3-4 non-hematologic toxicity was $26 \%$ and $27 \%$, respectively.

Conclusion: Preoperative chemo RT with chemotherapy for distal rectal cancer has clinical activity ( 3 of 25 pCR) and acceptable toxicity.
\end{abstract}

\section{Introduction}

Colorectal cancers among the 10 commonest cancers in India with $>33000$ cases diagnosed each year in the India. One-third of this number is contributed by rectal cancers [1]. Despite the high rate of curability with combined modality therapy, some patients experience significant treatment-associated morbidity, and other patients develop locoregional failure or distant metastasis. In addition to achieving cure, sphincter preservation is an important goal of therapy. Improvements in clinical outcome have been realized with wide acceptance of continuous infusion 5-fluorouracil (5-FU)-based neoadjuvant chemoradiotherapy (chemoRT) and the use of total mesorectal excision.

The results of large randomized trials comparing neoadjuvant pelvic radiotherapy (RT) alone versus RT plus concurrent 5-FU have demonstrated improvement in locoregional disease control with the addition of concurrent chemotherapy [2,3]. Attempts to improve on this approach have focused primarily on testing new agents added to the backbone of 5-FU plus RT to enhance the pathologic complete response (pCR) rate. Drugs with high activity in the metastatic disease setting have been of particular interest to apply to rectal cancer clinical studies. However, the integration of new RT techniques is also pertinent to this clinical research question. The Radiation Oncology Group (RTOG) 0012 study was a Phase II trial in which patients were randomly assigned to either hyperfractionated pelvic RT plus continuous infusion 5-FU or standard pelvic RT plus continuous infusion 5-FU and irinotecan [4]. That study was successful in that both arms achieved very high pCR rates, $26 \%$ in each arm. However, both arms were also associated with high rates of acute Grade 3 or greater toxicity ( $42 \%$ and $51 \%$, respectively, for each arm), and therefore, neither regimen was suitable for further development.

\section{Methods and Materials}

From January 2011 to June 2013, 25 patients with locally advanced (fixed tumor by digital rectal exam or T3-4 tumor by computed tomography [CT]/ sonography) rectal cancer who received CCRT were retrospectively reviewed. The neoadjuvant CCRT for locally advanced rectal cancer in our hospital was 5-fluorouracil $375 \mathrm{mg} / \mathrm{m} 2$ plus leucovorin $20 \mathrm{mg} / \mathrm{m} 2$ intravenously for 1 hour, weekly, with concurrent with radiotherapy (180cGy/ day, Monday- Friday, for 5 weeks). For radiotherapy planning image acquired using dedicated CT simulator with a slice thickness $2.5 \mathrm{~mm}$. then image transfer to Eclipse planning system. Clinical target volume (CTV) include thetumor bed and localregional lymph nodes. Planning target volume (PTV) generated by 


\section{Cancer Therapy \& Oncology International Journal}

giving margin of $1 \mathrm{~cm}$. Dose to PTV was 45Gy in 25 fraction 1.8 per fraction daily, 5 days a week, radiation portal includedanteriorposterior/posterior-anterior, with opposed lateral fields. Beam energies included6-MV, and 15-MV photons, patients underwent three-dimensional treatment planning.After completion of chemotherapy, patients underwent CT orPETCT and clinical evaluation for disease status. Patients underwent surgery 4 to 8 week after completion of CRT. Final histopathologynoted. 21 patients received adjuvant chemotherapy with FOLFOX 3 weekly total 6 cycles. End point of study was recurrence and or metastasis. Patients were followed every 3 months with physical examination as well as radiological examination include as clinically indicated. Response evaluation was done RESCIST criteria version 1.1.. For statistical analysis data was arranged SPSS 19 versions. Descriptive studies were done for parameter, survival, diseasefree survival, and local control rates for the entire cohort. The log-rank test was used to analyze prognostic factors for the entire cohort.Kaplan mair analysis used for survival analysis. P value $<0.05$ is considered as statically significant.

Table 1: Patient Characteristics.

\section{Results}

\section{Patients Characteristics}

Patient characteristics are summarized in (Table 1). Between January 2011 to June 2013, 105 patients of carcinoma rectum registered in our department. 25 patients had started on NACRT protocol, 25 patients non metastatic locally advancehad complete treatment NACRT, surgery and adjuvant chemotherapy wear available. The median age of the patients was 52.0 years. 23 patients were males and 2 patients wear females. The median duration of symptoms was six months. Bleeding per rectum was the most commonpresenting symptoms followed by anal discomfort, alteration of bowel habbit. Diarrhea and watery discharge.On histopathologicalall patients are Adenocarcinoma locally advance, non-metastasis.Treatment details (Table 2) Treatment modalities consisted of neo adjuvant chemo radiation then surgery and adjuvant chemotherapy. All patients underwent surgery, out of which 11patients underwent APR, 14 patients were underwent LAR.

\begin{tabular}{|c|c|}
\hline \multicolumn{2}{|c|}{ Patients characteristics } \\
\hline Total patients & 25 \\
\hline Male & $23(92 \%)$ \\
\hline Female & $02(8 \%)$ \\
\hline \multicolumn{2}{|l|}{ Age } \\
\hline Mean & 51.6 years \\
\hline Median & 52 years \\
\hline \multicolumn{2}{|l|}{ KPS } \\
\hline $70-80$ & $10(40 \%)$ \\
\hline$>80$ & $15(60 \%)$ \\
\hline \multicolumn{2}{|l|}{ Histopathology } \\
\hline Adenocarcinoma & $23(92 \%)$ \\
\hline Squamous cell carcinoma & $02(8 \%)$ \\
\hline \multicolumn{2}{|l|}{ Growth from anal canal } \\
\hline$<6 \mathrm{~cm}$ & $15(60 \%)$ \\
\hline$>6 \mathrm{~cm}$ & $10(40 \%)$ \\
\hline \multicolumn{2}{|l|}{ T stage (clinical) } \\
\hline T3 & $20(80 \%)$ \\
\hline $\mathrm{T} 4$ & $05(20 \%)$ \\
\hline \multicolumn{2}{|l|}{ N (stage clinical) } \\
\hline No & $05(20 \%)$ \\
\hline N1 & $06(24 \%)$ \\
\hline $\mathrm{N} 2$ & $08(32 \%)$ \\
\hline N3 & $04(16 \%)$ \\
\hline $\mathrm{Nx}$ & $02(8 \%)$ \\
\hline \multicolumn{2}{|l|}{ Ajcc stage } \\
\hline Stage IIA & \\
\hline
\end{tabular}




\section{Cancer Therapy \& Oncology International Journal}

\begin{tabular}{|l|l|}
\hline Stage IIB & \\
\hline Stage IIIB & \\
\hline Stage IIIC & \\
\hline
\end{tabular}

Table 2: Treatment Details.

\begin{tabular}{|c|c|}
\hline \multicolumn{2}{|c|}{ Treatment Details } \\
\hline Chemotherapy Regime & patients \\
\hline 5FU and Levucoverine & $23(92 \%)$ \\
\hline Cisplatin and 5FU & $02(8 \%)$ \\
\hline Chemotherapy cycles & $2(8 \%)$ \\
\hline 3 & $2(8 \%)$ \\
\hline 4 & $19(76 \%)$ \\
\hline 5 & $2(8 \%)$ \\
\hline 6 & $11(44 \%)$ \\
\hline Surgery & $14(56 \%)$ \\
\hline APR & \\
\hline LAR & $02(8 \%)$ \\
\hline Dose RT & $23(92 \%)$ \\
\hline $40 G y$ & \\
\hline 45 Gy & \\
\hline
\end{tabular}

\section{Treatment Toxicity and Complication}

There wear no surgical complications in form of post-operative death or wound complications in form of post operative death or wound complications. RT toxicity occurred in all patients in form of grade I and grade II dermatitis and there wear grade 3 in $7 \%$ of patients. All patient's complete treatment with no significant toxicity or treatment complication.

\section{Clinical outcome(Table 3)}

Table 3: Clinical Outcome.

\begin{tabular}{|c|c|}
\hline \multicolumn{2}{|c|}{ Clinical outcome } \\
\hline HPE after Surgery & Patients \\
\hline Adenocarcinoma & $20(80 \%)$ \\
\hline Squamous cell carcinoma & $02(08 \%)$ \\
\hline No residual Tumor & $03(12 \%)$ \\
\hline \multicolumn{2}{|l|}{ Margin } \\
\hline Positive & $03(12 \%)$ \\
\hline Negative & $22(88 \%)$ \\
\hline \multicolumn{2}{|l|}{ Lymph nodes } \\
\hline Positive & $06(24 \%)$ \\
\hline Negative & $19(76 \%)$ \\
\hline \multicolumn{2}{|l|}{ Status at last follow up } \\
\hline No evidence of disease & $18(72 \%)$ \\
\hline Diseased & $07(18 \%)$ \\
\hline \multicolumn{2}{|l|}{ Failure } \\
\hline Local & $04(16 \%)$ \\
\hline Distant & $03(12 \%)$ \\
\hline Both & $01(4 \%)$ \\
\hline
\end{tabular}




\section{Cancer Therapy \& Oncology International Journal}

25 evaluable patients, 3 had a pCR. Tumor down staging was observed in 10 (40\%) sphincter preservation were observed in $56 \%$ of patients, as demonstrated by the requirement for abdominoperineal resection (APR) in 44\%of patients. No significant association was observed between the pathologic factors ( $\mathrm{T}$ stage, $\mathrm{N}$ stage and margin status) but the use of APR had significant on overall survival $(0.026)$ distal tumors $(<6 \mathrm{~cm}$ from the anal verge) were more likely to require APR.

\section{Discussion}

The important goals of rectal cancer therapy include improvement of survival, local control, and sphincter preservation. For the development of new neoadjuvant approaches, overall survival is indisputably the reference standard study endpoint by which efficacy is measured. However, several studies have demonstrated that the pCR is predictive of other clinically relevant endpoints, including sphincter preservation, relapse-free survival, and a reduction in distant metastasis [5-9]. Moreover, the use of pCR appears to identify a favorable prognostic patient group that has an improved overall survival. Neoadjuvant rectal cancer trials have commonly adopted the use of the pCR as a reliable and meaningful intermediate endpoint. In the present study, we found that the combination of leucoverine and 5FU with RT results in a promising $\mathrm{pCR}$ rate with acceptable toxicity at the recommended doses. A direct comparison of the $\mathrm{pCR}$ rates from different studies has limited value but can be useful as a rough approximation of efficacy and for hypothesis generation. In this context, a general trend toward progressive improvement in pCR can be seen in clinical trials with newer neoadjuvant regimens for rectal cancer.

In two large multicenter Phase III studies (European Organization for Research and Treatment of Cancer 22921 and Federation Francophone de Cancerologie Digestive 9203), the rate of pCR for neoadjuvant 5-FU/leucovorin plus concurrent pelvic RT vs. RT alone was $11-14 \%$ and $4-5 \%$, respectively [3,10]. Four published studies that have examined the capecitabine plus pelvic RT demonstrated pCR rates in the range of 9-23\% (mean, 15\%) [11-14]. As the intensity of preoperative chemoRT is increased in an attempt to improve the rate of pCR, attention must also be placed on the toxicity of the therapy. In the preceding RTOG study, RTOG 0012, both study arms (5-FU plus hyperfractionated RT and 5-FU plus irinotecan plus standard RT) showed high rates of pCR (26\%); however, both arms were associated with high rates of acute toxicity of Grade 3 or greater, $42 \%$ and $51 \%$, respectively [4].

Radiation dose is of critical importance in down staging of cancer. The dose response of rectal cancer is steep in the dose range of 40 to $60 \mathrm{~Gy}$. Several studies have shown the impact of radiation dose escalation on the rate of pathological complete response to neoadjuvant therapy[15,16]. In a review of patients at Princess Margaret Hospital who received 40 Gy, 46 Gy, or 50 Gy in $2 \mathrm{~Gy} /$ fraction with continuous infusion 5-FU, the pathological complete response was $18 \%, 23 \%$, and $33 \%$ respectively for the three dose levels [17]. The two-year local relapse-free survival was $72 \%, 90 \%, 89 \%$ and disease-free survival $62 \%, 84 \%$, and $78 \%$ for the $40 \mathrm{~Gy}, 46 \mathrm{~Gy}$, and 50 Gy levels respectively [18]. The overall survival was $72 \%, 94 \%$, and $92 \%$ respectively. Doses of 46 Gy or 50 Gy were more effective than $40 \mathrm{~Gy}$, but there was no difference between 46 or 50 Gy. Similar results have been reported from other studies as well.

The definitive phase III study in favor of preoperative radiation therapy was the CAO/ARO/AIO-94 study performed by the German Rectal Cancer group [19]. Eight hundred twenty-three clinically staged T3 and T4 or node-positive rectal cancers were randomized to arm 1: Preoperative chemotherapy and radiation therapy followed by TME 6 weeks later, or arm 2: TME followed by postoperative chemotherapy and radiation therapy. The radiation therapy used was 50.4 Gy in 28 fractions with a 5.4 Gy as a small volume boost in the postoperative arm. The chemotherapy used was 5 -FU given as $1 \mathrm{~g} / \mathrm{m} 2$ per day during the 1 st and 5 th weeks of radiotherapy as a 120-hour continuous infusion. Both arms received four additional cycles of 5 -FU at $500 \mathrm{mg} / \mathrm{m} 2$ per day for 5 days every 4 weeks. All surgeons were trained in the use of TME and were asked prior to treatment to evaluate the possibility of sphincter preservation.

The 5-year results revealed a pelvic recurrence ratio of $6 \%$ versus $13 \%(p=0.02)$ in favor of the preoperative arm. The distant recurrence rate was $36 \%$ versus $38 \%$ ( $p=$ NSS), diseasefree survival was $68 \%$ versus $65 \%$ ( $p=$ NSS), and overall survival was $76 \%$ versus $74 \%$ ( $p=$ NSS) for preoperative radiation versus postoperative, respectively. There was significant tumor down staging after preoperative combined modality treatment with an $8 \%$ pathological complete response rate. Nodal positivity was $25 \%$ in the preoperative versus $40 \%$ in the postoperative arm. The sphincter preservation rate in 188 patients with low-lying tumors (declared by the surgeon prior to randomization to require an APR) revealed that $39 \%$ versus $19 \%$ had a sphincter-preserving low anterior resection $(\mathrm{p}=0.004)$ in the preoperative versus the postoperative arm. There were fewer acute $(27 \%$ vs. $40 \%)$ and late toxicities ( $14 \%$ vs. $24 \%)$ in preoperative-treatment group. Thus, preoperative combined preoperative chemotherapy and radiation therapy resulted in significantly less local failures in the pelvis by half and also provided twice the sphincter preservation. Importantly, there was no difference in overall survival or diseasefree survival between the two arms.

Clinical T4 tumors may not be resected completely due to tumor fixation. Preoperative radiation treatment is recommended to facilitate curative resections. M.D. Anderson investigators demonstrated that preoperative chemotherapy and radiation therapy increased overall survival ( $80 \%$ vs. $60 \%$ ), local control (95\% vs. $66 \%$ ), and the number of sphincter preserving procedures (35\% vs. $7 \%$ ) as compared to radiation alone [20]. Memorial Sloan-Kettering Cancer Center reported a gross total resection rate of $97 \%$, pathological complete response rate of $25 \%$, 4 -year local control of $70 \%$, and 4-year overall survival of $67 \%$ when giving preoperative chemotherapy of 5-FU and leucovorin with 50.4 Gy of radiation followed by surgery [21]. Preoperative 


\section{Cancer Therapy \& Oncology International Journal}

radiation and chemotherapy resulted in improved resectability rates and possible improved local control and survival.

The IORT experience at MGH was reviewed by Nakfoor et al. [22]. Preoperative continuous infusion 5-FU plus 50.4 to 54 Gy of radiation was given followed by a 4 - to 6 -week break and surgery. No intraoperative radiation was given if metastases were present at surgical exploration, if there were adequate margins $>1 \mathrm{~cm}$, or if there was less than T4 disease. Ten to 12.5 Gy were given for complete resection, 12.5 to 15 Gy for microscopic residual, and 17.5 to $20 \mathrm{~Gy}$ for gross residual disease. The 5-year local control was $90 \%, 65 \%, 55 \%$, and the disease specific survival at 5 years was $65 \%, 45 \%$, and $15 \%$, for these three dose levels, respectively. The 5-year actuarial risk of complications was $15 \%$, however. The risk of peripheral neuropathy was $20 \%$ for doses $>15$ Gy. IORT improves local control, especially with a gross total resection, but not survival for locally advanced rectal cancer.

\section{Conclusion}

We have shown that neoadjuvant pelvic RT with 5FUand leucoverine for clinical Stage T3 and T4 rectal cancer is associated with manageable toxicity and yields a hpCR rate. Although the results of the RTOG 0247 trial are encouraging, they also highlight the need to identify more effective and less toxic regimens, through incorporation of new radiation sensitizers, novel methods of RT delivery, and the selection of patients according to molecular classifiers.

\section{References}

1. Parkin DM, Bray F, Ferlay J, Pisani P (2005) Global cancer statistics, 2002. CA Cancer J Clin 55(2): 74-108.

2. Bosset JF, Collette L, Calais G, Mineur L, Maingon P, et al. (2006) Chemotherapy with preoperative radiotherapy in rectal cancer. $\mathrm{N}$ Engl J Med 355(11): 1114-1123.

3. Gerard JP, Conroy T, Bonnetain F, Bouché O, Chapet O, et al. (2006) Preoperative radiotherapy with or without concurrent fluorouracil and leucovorin in T3-4 rectal cancers: Results of FFCD 9203. J Clin Oncol 24(28): 4620-4625.

4. Mohiuddin M, Winter K, Mitchell E, Hanna N, Yuen A, et al. (2006) Randomized phaseII study of neoadjuvant combined-modality chemoradiation fordistal rectal cancer: Radiation Therapy Oncology Group trial 0012. J Clin Oncol 24(4): 650-655.

5. Stipa F, Chessin DB, Shia J, Paty PB, Weiser M, et al. (2006) A pathologic complete response of rectal cancer to preoperative combined-modality therapy results in improved oncological outcome compared with those who achieve no downstaging on the basis of preoperative endorectal ultrasonography. Ann Surg Oncol 13(8): 1047-1053.

6. Collette L, Bosset JF, den Dulk M, Nguyen F, Mineur L, et al. (2007) Patients with curative resection of cT3-4 rectal cancer after preoperative radiotherapy or radiochemotherapy: Does anybody benefit from adjuvantfluorouracil-based chemotherapy? A trial of the European Organisation for Research and Treatment of Cancer Radiation Oncology Group. J Clin Oncol 25(28): 4379-4386.

7. Garcia-Aguilar J, Hernandez de Anda E, Sirivongs P, Lee SH, Madoff $\mathrm{RD}$, et al. (2003) A pathologic complete response to preoperative chemoradiation is associated with lower local recurrence and improved survival in rectal cancer patients treated by mesorectal excision. Dis Colon Rectum 46(3): 298-304.
8. Vecchio FM, Valentini V, Minsky BD, Padula GD, Venkatraman ES, et al. (2005) The relationship of pathologic tumor regression grade (TRG) and outcomes after preoperative therapy in rectal cancer. Int J Radiat Oncol Biol Phys 62(3): 752-760.

9. Capirci C, Valentini V, Cionini L, De Paoli A, Rodel C, et al. Prognostic value ofpathologic complete response after neoadjuvant therapy inlocally advanced rectal cancer: Long-term analysis of 566 ypCR patients. Int J Radiat Oncol Biol Phys 72(1): 99-107.

10. Bosset JF, Collette L, Calais G, Mineur L, Maingon P, et al. (2006) Chemotherapy with preoperative radiotherapy in rectal cancer. $\mathrm{N}$ Engl J Med 355(11): 1114-1123.

11. Korkolis DP, Boskos CS, Plataniotis GD, Emmanuel Gontikakis, Ioannis j Karaitianos, et al. (2007) Pre-operative chemoradiotherapy with oral capecitabine in locally advanced, resectable rectal cancer. Anticancer Res 27(1B): 541-545.

12. Krishnan S, Janjan NA, Skibber JM, Rodriguez-Bigas MA, Wolff RA, et al. Phase II study of capecitabine (Xeloda) and concomitant boost radiotherapy in patients with locally advanced rectal cancer. Int J Radiat Oncol Biol Phys 66(3): 762-771.

13. Desai SP, El-Rayes BF, Ben-Josef E, Greenson JK, Knol JA, et al. A phase II study of preoperative capecitabine and radiation therapy in patients with rectal cancer. Am J Clin Oncol 30(4): 340-345.

14. Craven I, Crellin A, Cooper R, Melcher A, Byrne P, et al. (2007) Preoperative radiotherapy combined with 5 days per week capecitabine chemotherapy in locally advanced rectal cancer. Br J Cancer 97(10): 1333-1337.

15. Allal AS, Bieri S, Brundler MA, Soravia C, Gertsch P, et al. (2002) Preoperative hyperfractionated radiotherapy for locally advanced rectal cancers: A phase I-II trial. Int J Radiat Oncol Biol Phys 54(4): 1076-1081.

16. Movas B, Hanlon AL, Lanciano R, Scher RM, Weiner LM, et al. (1998) Phase I dose escalating trial of hyperfractionated pre-operative chemoradiation for locally advanced rectal cancer. Int J Radiat Oncol Biol Phys 42(1): 43-50.

17. Mohiuddin M, Winter K, Mitchell E, Hanna N, Yuen A, et al. (2006) Randomized phase II study of neoadjuvant combined-modality chemoradiation for distal rectal cancer: Radiation Therapy Oncology Group trial 0012. J Clin Oncol 24(4): 650-655.

18. Wiltshire KL, Ward IG, Swallow C, Oza AM, Cummings B, et al. (2006) Preoperative radiation with concurrent chemotherapy for resectable rectal cancer: Effect of dose escalation on pathologic complete response, local recurrence-free survival, disease-free survival, and overall survival. Int J Radiat Oncol Biol Phys 64(3): 709-716.

19. Sauer R, Becker H, Hohenberger W, Hess C, Becker H, et al. (2004) Preoperative versus postoperative chemoradiotherapy for rectal cancer. N Engl J Med 351: 1731-1740.

20. Weinstein GD, Rich TA, Shumate CR, Skibber JM, Cleary KR, et al. (1995) Preoperative infusional chemoradiation and surgery with or without an electron beam intraoperative boost for advanced primary rectal cancer. Int J Radiat Oncol Biol Phys 32(1): 197-204.

21. Minsky BD, Cohen AM, Kemeny N, Enker WE, Kelsen DP, et al. (1992) Enhancement of radiation induced downstaging of rectal cancer by fluorouracil and high dose leucovorin chemotherapy. J Clin Oncol 10(1): 79-84.

22. Nakfoor BM, Willett CG, Shellito PC, Kaufman DS, Daly WJ (1998) The impact of 5-fluorouracil and intraoperative electron beam radiation therapy on the outcome of patients with locally advanced primary rectal and rectosigmoid cancer. Ann Surg 228(2): 194-200. 


\section{Your next submission with Juniper Publishers will reach you the below assets}

- Quality Editorial service

- Swift Peer Review

- Reprints availability

- E-prints Service

- Manuscript Podcast for convenient understanding

- Global attainment for your research

- Manuscript accessibility in different formats ( Pdf, E-pub, Full Text, Audio)

- Unceasing customer service

Track the below URL for one-step submission https://juniperpublishers.com/online-submission.php 